
la Grande Guerre

\title{
Dynamique de la végétation sur le site de la Cote 108 et dans la forêt domaniale de Sapigneul
}

\section{Emilie Gallet-Moron, Jérôme Buridant et Laurent Chalumeau}

\section{OpenEdition}

\section{Journals}

Édition électronique

URL : https://journals.openedition.org/geohist/633

DOI : $10.4000 /$ geohist.633

ISSN : 2264-2617

Éditeur

Association française de la Revue de géographie historique

Référence électronique

Emilie Gallet-Moron, Jérôme Buridant et Laurent Chalumeau, « Dynamique de la végétation sur le site de la Cote 108 et dans la forêt domaniale de Sapigneul », Revue de géographie historique [En ligne], 14-15 | 2019, mis en ligne le 28 mai 2018, consulté le 12 juin 2021. URL : http:// journals.openedition.org/geohist/633 ; DOI : https://doi.org/10.4000/geohist.633

Ce document a été généré automatiquement le 12 juin 2021.

Ce(tte) œuvre est mise à disposition selon les termes de la Licence Creative Commons Attribution Pas d'Utilisation Commerciale - Pas de Modification 4.0 International. 


\title{
Dynamique de la végétation sur le site de la Cote 108 et dans la forêt domaniale de Sapigneul
}

\author{
Emilie Gallet-Moron, Jérôme Buridant et Laurent Chalumeau
}

\section{Introduction}

Outre l'énorme bilan humain, la Première Guerre mondiale a également fortement et durablement marqué les paysages. Sur le front, le pilonnage de l'artillerie et la guerre des mines ont engendré la destruction de la végétation et des sols. La destruction des terres agricoles et la présence de nombreux obus et de munitions non explosés enfouis ont conduit au classement dès 1919 d'environ 120000 hectares du champ de bataille français en zones rouges, interdisant ou limitant les activités possibles.

Dans ce contexte, le projet exploratoire présenté ici s'intéresse à la recolonisation de la végétation en zone dévastée, sur le site de la Cote 108 (Aisne et Marne). La première partie de l'étude a pour objectif de reconstituer la dynamique de recolonisation de la végétation dans le temps, par photo-interprétation. La seconde partie s'attache quant à elle, à décrire le sol de la zone d'étude et à quantifier les macro-restes métalliques qui subsistent cent ans après la fin du conflit. Ces informations permettent ensuite de proposer des hypothèses susceptibles d'expliquer les vitesses de recolonisation étudiées précédemment.

\section{Présentation du site d'étude : contexte historique et géographique}

3 Le site d'environ 180 hectares étudié ici, est celui de la " Montagne de Sapigneul », plus communément appelé depuis 1914 "Cote 108 ». Site incontournable de la Grande Guerre, il se trouve à l'interface des départements de l'Aisne (commune de Berry-auBac) et de la Marne (commune de Cormicy). Site d'interfluve entre l'Aisne au nord et la 
Loivre au sud, il est à l'est de la confluence entre l'Aisne et le canal de l'Aisne à la Marne (Fig. 1a). Ce site stratégique durant la guerre s'étend sur une croupe de craie blanche du Sénonien (Crétacé supérieur) et de colluvions sableuses remaniées (Le Roux et Allouc, 1988 ; Fig. 1b). Les hauteurs, d'une altitude maximale de $93 \mathrm{~m}$, dominent la vallée de l'Aisne d'une quarantaine de mètres. Elles commandent le passage de l'Aisne et la jonction du canal latéral à l'Aisne et de celui de la Marne à l'Aisne, qui constituent autant de coupures pour les armées. Ce relief sert de point d'appui aux réseaux de défense du front de 1914 à 1918, dans la continuité du Chemin des Dames dont il constitue la terminaison orientale. Marquant le front entre les lignes françaises et allemandes, il est le théâtre d'une importante guerre de mines dans sa partie ouest et d'un intense pilonnage dans sa partie est. À l'issue de la Première Guerre mondiale, le sol est profondément marqué par les tranchées, les entonnoirs de mines, ainsi que par les multiples trous d'obus. Outre des entonnoirs toujours visibles sur le terrain, notamment le plus gros qui mesure $40 \mathrm{~m}$ de diamètre et $80 \mathrm{~m}$ de profondeur, l'imagerie LiDAR permet de se rendre compte de l'ampleur des bouleversements existant dans la zone. A titre d'exemple, Taborelli et ses collaborateurs (2017) ont dénombré sur la partie occidentale du site (Cote 108), à l'aide de l'image LiDAR, 607 impacts d'obus avec des densités qui s'échelonnent entre 0 et 54 impacts par hectare (Fig. 1d).

Document 1 : (a) Carte de localisation de la zone d'étude (BD TOPO, IGN - BD FORET v2, IGN), (b) Carte géologique de la zone d'étude (BRGM), (c) Carte d'Etat-Major (1825), (d) Visualisation des bouleversements de la Grande Guerre sur l'image LIDAR (ombrage $315^{\circ}$ combiné avec le LRM) (LiDAR réalisé dans le cadre du projet IMPACT 14-18).
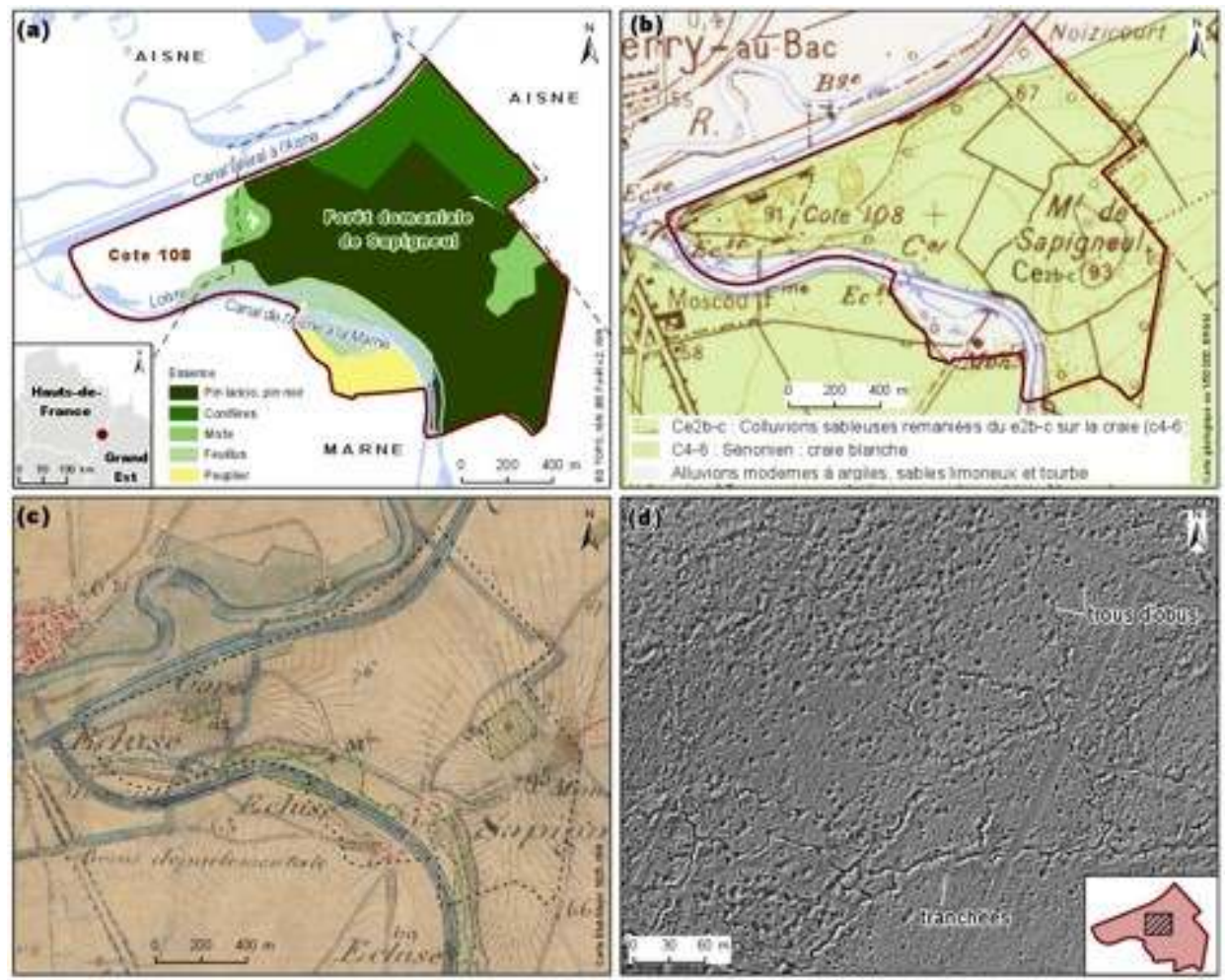

La carte d'État-Major permet de constater qu'en 1825, le site était majoritairement composé de terres agricoles et que les zones boisées se limitaient à quelques ripisylves le long des canaux et des cours d'eau au nord et au sud-ouest de la zone d'étude et à une petite zone boisée de moins de 4 ha à l'est de la zone (Fig. 1c). Suite au conflit, la 
végétation a complètement disparu et le site est classé en zone rouge. Certaines terres de la zone rouge, impossible à remettre en culture, ont été confiées à l'Administration des Eaux et Forêts qui a ainsi créé 11 nouvelles forêts comme celles de Verdun (9214 ha), des Éparges (660 ha), de Vauclerc (717 ha) ainsi que celle de Sapigneul (115 ha) (Decencière Ferrandière, 1960; Amat, 1987). Ces anciennes terres agricoles devenues forêts ont été plantées majoritairement de résineux (Pin laricio et Pin noir étant les essences dominantes) et ceci de façon échelonnée à partir du milieu des années 1920. Le site d'étude offre alors un contraste intéressant entre une partie plantée et gérée par l'ONF (Office National des Forêts) aujourd'hui (zone est, forêt domaniale de Sapigneul) et une partie ouest (site de la Cote 108), propriété privée où la recolonisation de la végétation se fait plus spontanément.

\section{Matériel et méthode}

\section{A. Analyse de la recolonisation de la végétation}

$5 \quad$ L'analyse de la recolonisation de la végétation est réalisée par photo-interprétation. Six séries de photographies aériennes (IGN) en période de végétation ont été sélectionnées : 1929, 1949, 1957, 1969, 1990, 2016 (BD ORTHO HR, IGN 2016). Initialement, un pas de temps d'environ 20 ans a été choisi mais une période charnière s'est dessinée entre 1949 et 1969, ce qui a justifiée l'ajout d'une série de photographies de 1957. Les photographies aériennes, de 1929 à 1990, ont été géoréférencées dans un Système d'Information Géographique.

Une grille composée de mailles de $50 \mathrm{~m}$ x $50 \mathrm{~m}$ a été créée sur la zone d'étude afin de relever pour chaque maille le pourcentage d'occupation du sol de chaque catégorie présente. Cinq grandes catégories d'occupation du sol ont été retenues: sol nu, végétation herbacée et arbustive, végétation arborée, bâti et occupation humaine, eau (Fig. 2).

Document 2:Visualisation des types d'occupation du sol «sol nu», "végétation herbacée-arbustive " et "végétation arborée » dans un pixel sur les photographies 


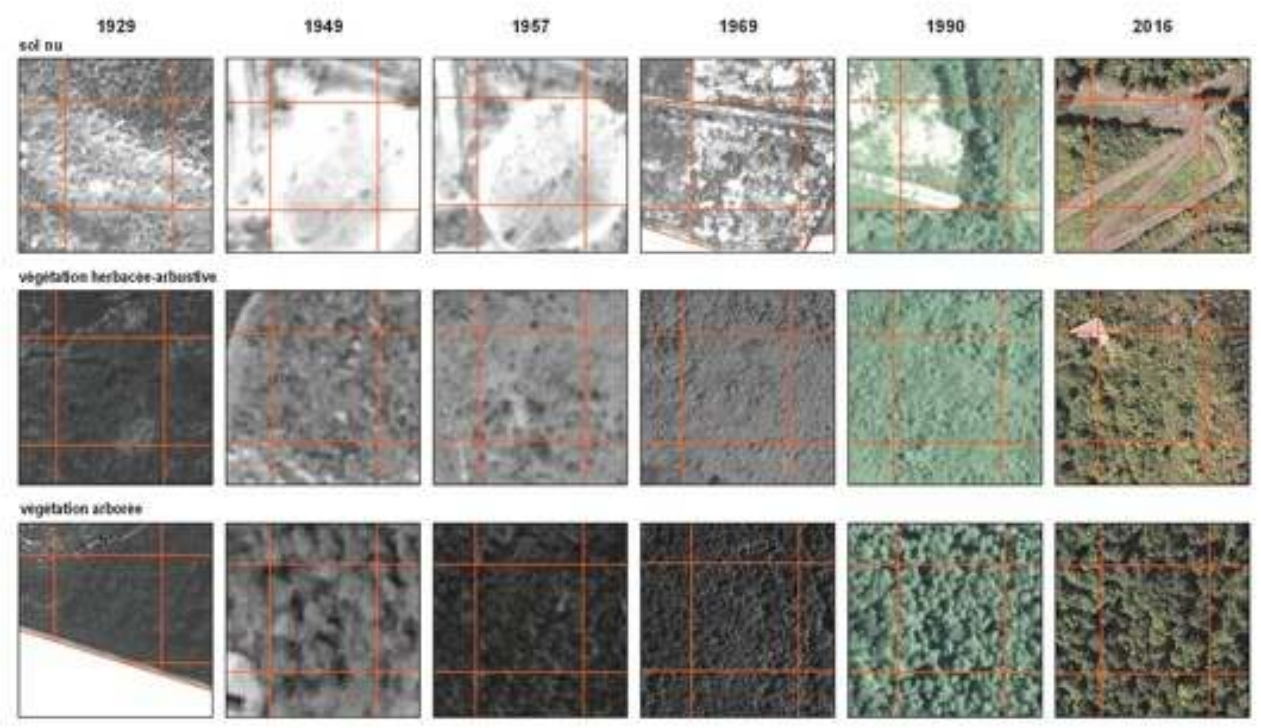

7 Toute l'analyse spatiale a été réalisée sous ArcGIS Pro 2.0 (ESRI).

\section{B. Analyse des éléments du sol} actuellement. Dans ce contexte une fosse de $60 \mathrm{~cm}$ x $60 \mathrm{~cm}$ et de $45 \mathrm{~cm}$ de profondeur (profondeur jusqu'à la roche mère) a été creusée et un prélèvement d'un volume de 5 seaux de $20 \mathrm{~L}$ soit $100 \mathrm{~L}$ a été réalisé. Le second sol observé se situe entre deux trous d'obus. Afin de l'observer, une fosse de $50 \mathrm{~cm}$ x $50 \mathrm{~cm}$ et de $60 \mathrm{~cm}$ de profondeur a été creusée et 7 seaux de $20 \mathrm{~L}$ soit $140 \mathrm{~L}$ ont été prélevés.

Une fois décrits et la présence ou l'absence d'éléments métalliques notée, des prélèvements ont été réalisés. Pour avoir une idée de la quantité de macro-restes métalliques, c'est-à-dire de taille supérieure à $2 \mathrm{~mm}$, présents dans le sol, et limiter la quantité d'échantillons à analyser en laboratoire, un tamisage à $2 \mathrm{~mm}$ a été réalisé sur le terrain. L'intégralité des fractions grossières supérieures à $2 \mathrm{~mm}$ a été conservée alors que seule une partie de la fraction inférieure à $2 \mathrm{~mm}$ a été prélevée. En laboratoire, l'ensemble des prélèvements a ensuite fait l'objet d'un tamisage humide avec des tamis de $2 \mathrm{~mm}, 1 \mathrm{~mm}, 500 \mu \mathrm{m}$ et $250 \mu \mathrm{m}$ après défloculation. Après séchage, les éléments métalliques de chaque fraction granulométrique ont été extraits via un tri visuel et un aimant. La fraction globale ainsi que les macro-restes métalliques de chaque refus de tamis ont été pesés. 


\section{Résultats}

\section{A. Analyse de la recolonisation de la végétation}

11 La recolonisation de la végétation s'est déroulée progressivement et de manière contrastée entre la forêt domaniale et la Cote 108.

Il est intéressant de noter que dès 1929, sur le site de la Cote 108, la recolonisation de la végétation apparait sur la ripisylve de la Loivre, qui est un espace très favorable du fait de la présence de l'eau, d'une banque de graines dans le sol et d'une ancienne ambiance forestière. La continuité de ce phénomène se retrouve logiquement dans la forêt domaniale, où le boisement de cette fine bande de terre le long de la rivière a déjà commencé en 1929 avant de s'accentuer en 1949 (Fig. 4).

Par contre la recolonisation de l'ensemble de la zone de la Cote 108 par une végétation spontanée est très lente. En effet, sur les 118 pixels de la zone de la Cote 108, 46 ont un taux de sol nu supérieur ou égal à 50 \% en 1929, 45 en 1949, 20 en 1957 et 5 en 1969 (Fig. 3). En 1990 et 2016 les quelques pixels de sols nus restants le sont suite à des travaux. Ainsi le premier déclin de cette zone de sol nu n'intervient qu'en 1957 soit 40 ans après la fin du conflit. On remarque une inertie considérable dans la zone du gros cratère qui, jusqu'en 1969, est la zone regroupant les derniers pixels de sol nu. Aujourd'hui la zone de la Cote 108 est entièrement végétalisée par des arbustes et des épineux (Taborelli et al., 2017).

\section{Document 3 : Carte de la part de sol nu dans chaque pixel de $50 \mathrm{~m}$ x $50 \mathrm{~m}$ à chaque date.}
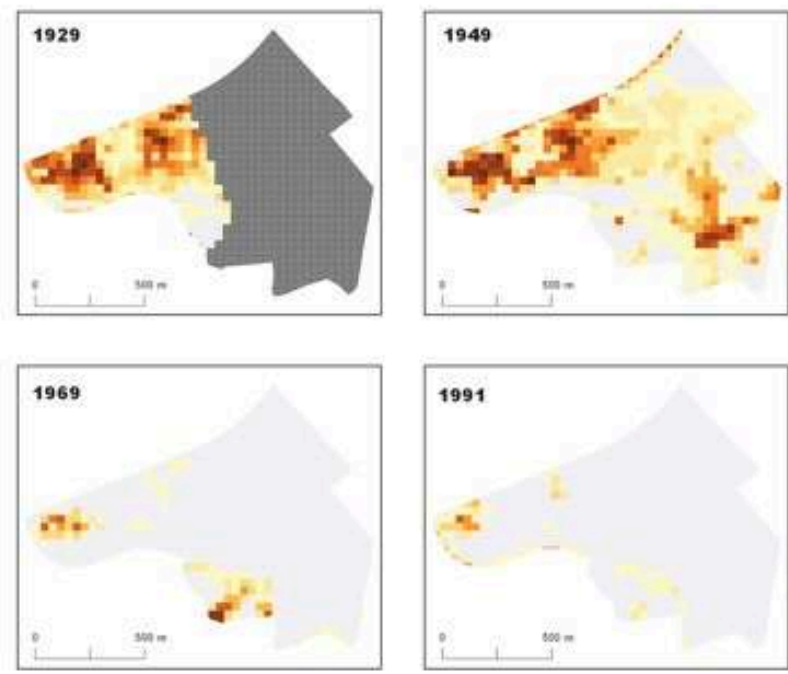
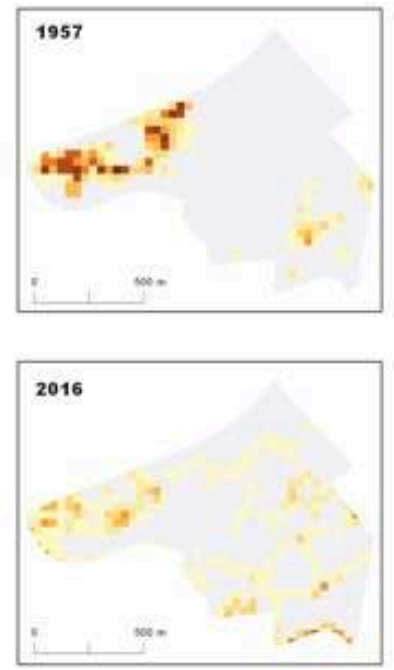

* de sol nu dans un pixel de soxsom

(2)

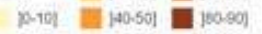

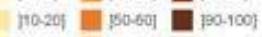

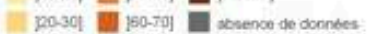


Document 4 : Carte de la part de la végétation arborée dans chaque pixel de $50 \mathrm{~m} \times 50 \mathrm{~m}$ à chaque date.
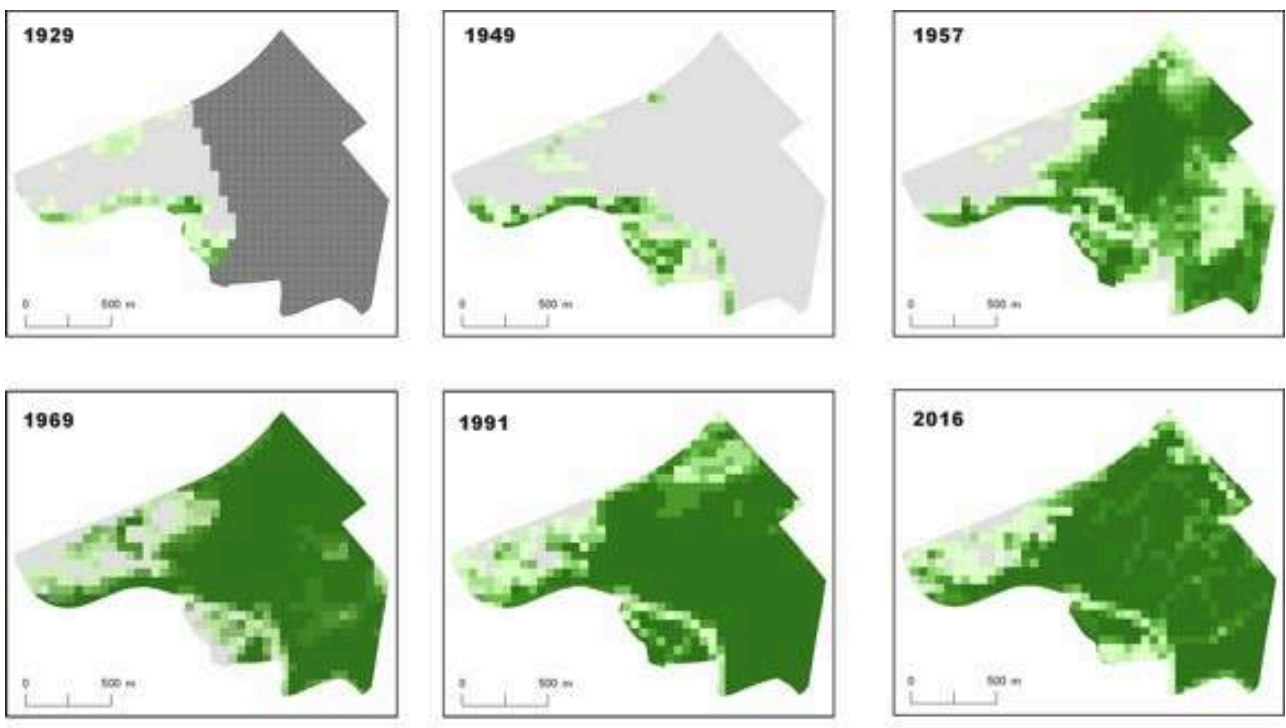

46 de surtace arborbe dans un pixel de $50 \times 50 \mathrm{~m}$

- 대 130-40 둘 $70-801$

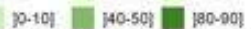

110-201

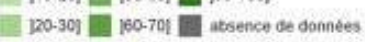

Concernant la forêt domaniale, la partie principale au nord du canal de l'Aisne à la Marne se distingue de la petite zone au sud du canal. En effet, cette dernière a subi des perturbations liées à la guerre nettement moins importantes (pas de tranchées, pas d'entonnoirs et peu de trous d'obus). De plus, le sol n'y est pas crayeux, mais composé d'alluvions modernes (Fig. 1b), et des peupliers et des feuillus y ont été plantés. De fait, cette zone est déjà en partie arborée en 1949, ce qui est loin d'être le cas dans la partie nord. Les premières coupes sont mêmes observables sur les photographies de 1969. Dans la partie au nord du canal, outre le cas particulier de la ripisylve, la majorité de la forêt domaniale est encore à l'état herbacé ou arbustif en 1949 malgré les plantations de conifères (Pin laricio et le Pin noir, Fig. 1a), effectuées dans les années 1930 (Fig. 5). Certains espaces à l'ouest et au sud de la zone présentent même une majorité de sol nu (Fig. 3). La photographie aérienne de 1957, montre une nette régression du sol nu dans cette zone, mais il faut attendre la photographie de 1969 pour le voir totalement disparaitre (Fig. 3). Côté plantations, elles n'évoluent pas uniformément sur la zone, certains espaces semblent en retard et/ou en échec (Fig. 4). C'est seulement en 1969, soit 50 ans après la fin du conflit et au moins 30 ans après les premières plantations, que le boisement semble s'uniformiser au sein de la forêt domaniale. C'est en 1991 que l'on assiste aux premières coupes dans la partie nord de la forêt domaniale. 
Document 5 : Carte à chaque date de l'occupation du sol par type majoritaire dans chacun des pixels.
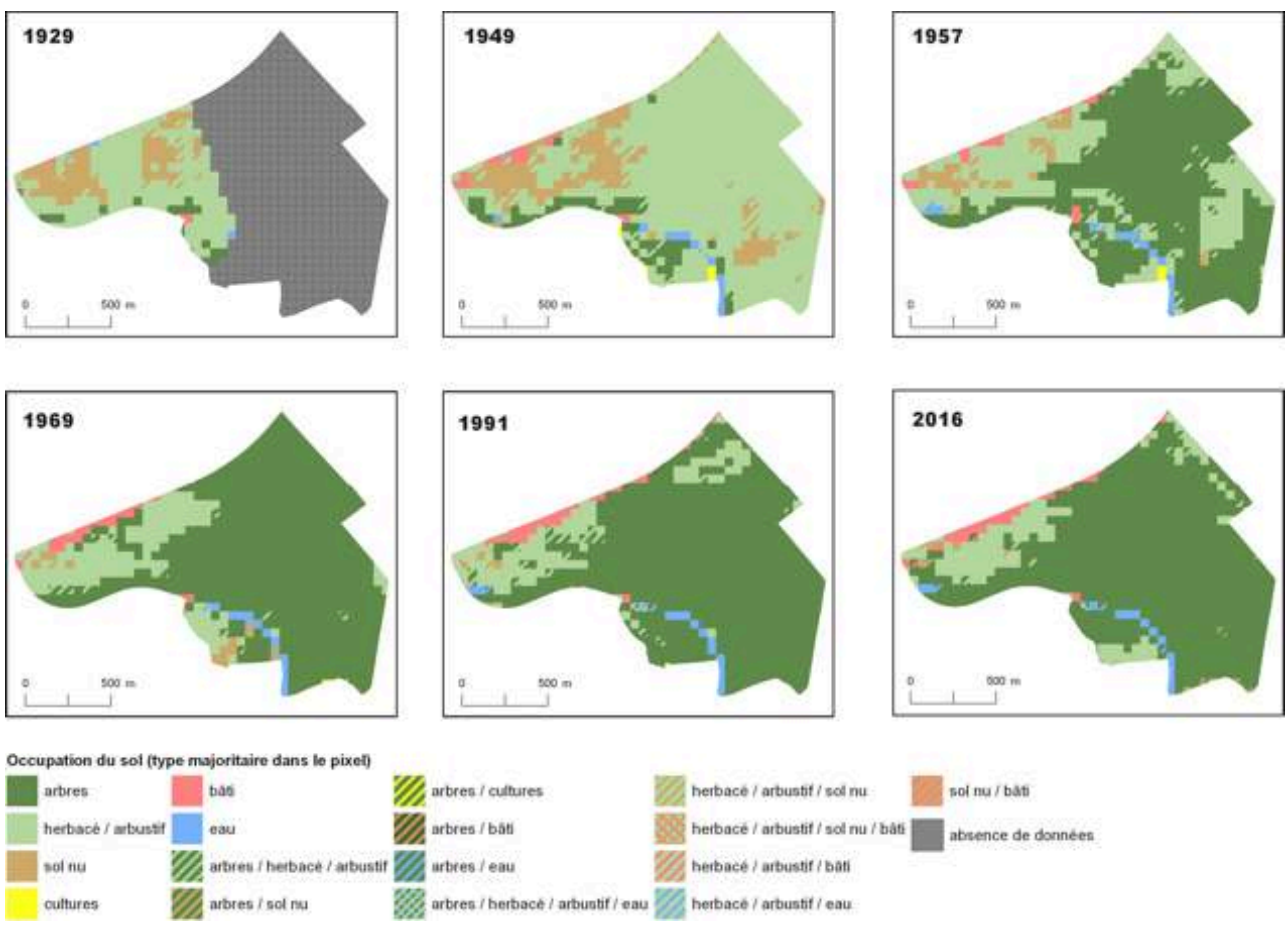

Finalement il aura fallu pratiquement un siècle pour assister à une recolonisation quasi-totale du site d'étude par la végétation.

\section{B. Analyse des éléments du sol}

Le sol observé au fond du trou d'obus présente $8 \mathrm{~cm}$ d'humus qui recouvrent $21 \mathrm{~cm}$ d'un horizon A limoneux très riche en fragments de roche mère. Cette dernière apparait ensuite très dégradée sur $10 \mathrm{~cm}$ puis fracturée (Fig.6). Des racines traversent l'ensemble du profil. De nombreux fragments métalliques de plus ou moins grande taille sont observables comme un fil lisse, un morceau d'obus et des balles de shrapnel. 
Document 6 : Photographie 1) du sol observé au fond du trou d'obus et quelques éléments métalliques trouvés dedans : 2) balle de shrapnel et 3) fragment d'obus.

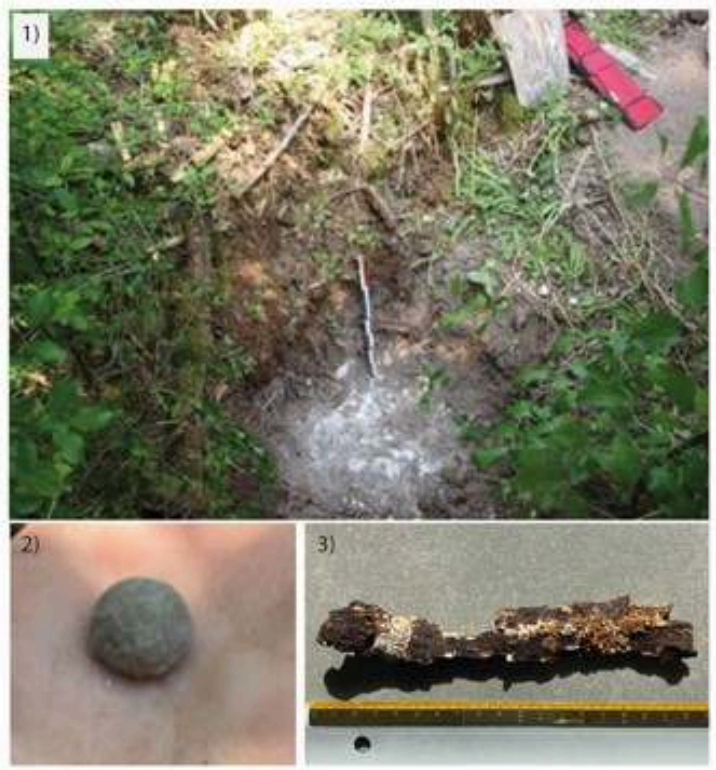

Le sol observé entre les trous d'obus ne présente quant à lui ni litière, ni humus (Fig. 7). Hormis quelques aiguilles de pin en surface, il ne subsiste pas de matière organique. Ainsi, il est composé d'un horizon limono-argileux très riche en fragments grossiers de craie qui repose sur $5 \mathrm{~cm}$ de craie fracturée sous laquelle se retrouve un niveau proche de l'horizon supérieur sur au moins $10 \mathrm{~cm}$. Ceci marque bien un démantèlement du sol et un mélange des horizons. Les racines sont ici présentes uniquement sur les $40 \mathrm{~cm}$ de supérieurs. Là aussi, de nombreux fragments métalliques sont observables comme un clou et des balles de shrapnel. 
Document 7 : Photographies 1) du sol observé entre des trous d'obus et quelques éléments métalliques trouvés dedans : 2) et 3 ) fragments de fusées d'obus.
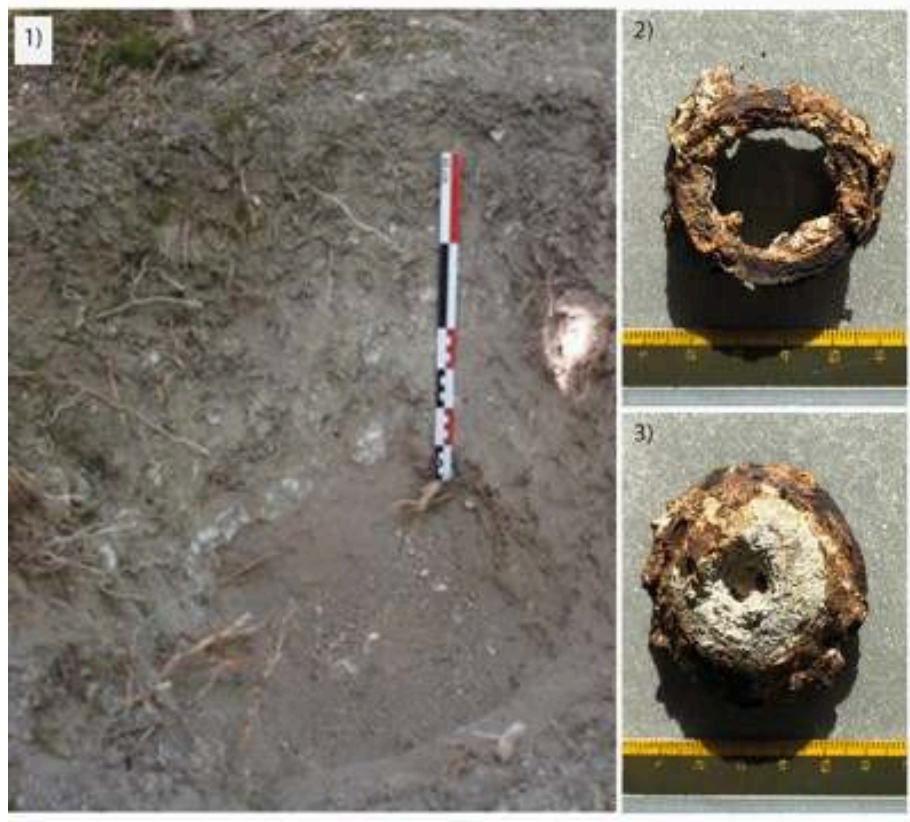

Le traitement en laboratoire du prélèvement effectué au fond du trou d'obus (tamisage et tri des éléments par observation et à l'aide d'un aimant), fait apparaître un peu plus de $1,23 \mathrm{~kg}$ de restes métalliques de taille supérieure à $125 \mu \mathrm{m}$ (Tab. 1). Bien que l'extrapolation de cette seule valeur soit périlleuse, elle permet de mieux se rendre compte de ce qu'elle représente soit un peu plus de 33 tonnes de macro-restes métalliques / hectare sur une profondeur de $50 \mathrm{~cm}$, ce qui est considérable. D'un point de vue répartition, les éléments métalliques représentent $15 \%$ de la fraction supérieure à $2 \mathrm{~mm}$. On peut aussi noter la part importante des fractions métalliques grossières, au regard des fractions fines ou fragmentées.

Tab. 1 : Résultats après tamisage et tri du prélèvement effectué au fond du trou d'obus.

\begin{tabular}{|l|l|l|l|}
\hline $\begin{array}{l}\text { Fractions } \\
\text { granulométriques }\end{array}$ & $\begin{array}{l}\text { Poids fraction } \\
\text { globale }(\mathrm{g})\end{array}$ & $\begin{array}{l}\text { Poids éléments } \\
\text { métalliques }(\mathrm{g})\end{array}$ & $\begin{array}{l}\text { Pourcentage } \\
\text { éléments } \\
\text { métalliques }(\%)\end{array}$ \\
\hline$>2 \mathrm{~mm}$ & 8359,49 & 1222,33 & 14,63 \\
\hline $1 \mathrm{~mm}-2 \mathrm{~mm}$ & 1185,83 & 4,64 & 0,39 \\
\hline $500 \mu \mathrm{m}-1 \mathrm{~mm}$ & 541,41 & 2,3 & 0,42 \\
\hline $250 \mu \mathrm{m}-500 \mu \mathrm{m}$ & 378,41 & 1,44 & 0,38 \\
\hline Total & & 1230,71 & \\
\hline
\end{tabular}

\section{Discussion}

Suite aux résultats obtenus, ce projet exploratoire amène à se demander quels peuvent être les facteurs explicatifs de cette inertie dans la recolonisation de la végétation et 
ceci même en cas de plantation. Il existe a priori des facteurs liés directement aux sols et d'autres à la végétation.

18 Au niveau des sols, plusieurs causes peuvent être avancées. Il ne faut pas oublier que notre site d'étude se trouve sur un sol crayeux qui est un sol drainant et chimiquement pauvre ce qui ne favorise pas a priori le développement de la végétation. Les bouleversements liés à la Première Guerre mondiale (tranchées, bombturbation, guerre de mines...), ont conduit à un mélange des horizons du sol voire à une absence de sol en annulant en partie les effets de pédogenèse, avec un sol en mosaïque. La végétation doit ainsi repousser sur des sols très peu évolués et très jeunes où toute la matière organique a disparu. Il est intéressant de noter que dans une étude menée sur la forêt domaniale du Mort-Homme qui, comme la forêt domaniale de Sapigneul, a été plantée sur des terres agricoles après la Première Guerre mondiale, certaines parcelles ont des peuplements denses et une croissance exceptionnelle (Amat, 1987). Mais ces peuplements ont poussé sur d'anciens sols agricoles profonds, sur limons épais et calcaires marneux, qui sont des sols bien plus riches et favorables que les sols crayeux de la forêt de Sapigneul. Il faut aussi noter que la mise à nu du sol a favorisé le ruissellement et l'érosion, notamment sur les surfaces en pente, retardant d'autant la pédogenèse (Doerr et al., 2000 ; 2006). L'hydrologie du sol a pu aussi être impactée. Si le creusement de sape a pu modifier les écoulements en profondeur et la drainance, la fracturation de la roche sous l'effet des bombardements va impacter l'hydrologie du sol et par ricochet la végétation. Hupy et Schaetzl (2006) ont montré l'accumulation de matière organique au fond de cratères de bombardements sur des sols calcicoles. En effet, pour eux, le bombardement a certes évacué une partie du sol mais il a surtout compacté le fond de fosse, permettant une humidité plus élevée, le développement de plantes et de fait l'épaississement des horizons $O$ et A. Nos observations sur substrat crayeux montrent un fonctionnement tout à fait différent, avec une fracturation de craie très importante en surface, une quasi-absence de strate herbacée et d'horizon 0. De fait, l'infiltration et la percolation sont dans notre cas favorisés, engendrant un taux d'humidité dans le sol très faible.

19 De plus, l'aspect pollution des sols n'est pas à négliger. Cette dernière peut être liée à de nombreux éléments qui vont réagir différemment en fonction de leur nature et des propriétés physiques et chimiques des sols. Les objets métalliques, comme ceux quantifiés dans cette étude, vont avoir un temps de résidence relativement important dans le sol. Certains éléments comme le fer (observé via le fil de fer en acier, le clou ou les restes d'obus) ne semblent pas influencer la croissance des plantes, même en grande concentration. D'autres comme le plomb (présent dans les balles d'armes à feu et dans les balles de shrapnel par exemple), vont avoir un comportement chimique et minéralogique qui se termine soit par l'absorption par les plantes, soit par la précipitation en minéraux insolubles (Jorgensen et Willems, 1987 ; Manninen et Tanskanen, 1993 ; Lin et al., 1995). D'autres encore, comme le chrome, l'arsenic, le cadmium, le cuivre (présent dans les balles, au niveau des ceintures et des fusées d'obus trouvés sur le terrain), le mercure, le nickel et le zinc sont souvent libérés progressivement dans sol à partir de résidus d'armes ou de munitions (Vasarevičius et Greičiute, 2004 ; Johnson et al., 2006 ; Lewis et al., 2010 ; Ghanbarizadeh et Nejad, 2012 ; Bausinger etal., 2007). Ces auteurs montrent que ces polluants - notamment l'arsenic et les métaux lourds - ont un impact sur la végétation, puisque seules les plantes résistantes vont arriver à se développer. 
tôté végétation, la difficulté de recolonisation est, sans doute, en partie due à une banque de graines et des restes racinaires limités car avant la Première Guerre mondiale, la majorité de la zone était occupée par des terres agricoles. D'ailleurs nos résultats montrent parfaitement l'impact positif de l'ambiance forestière puisque dans notre site d'étude, seule la ripisylve a une reprise spontanée et rapide de la végétation.

\section{Conclusion}

En un siècle la végétation semble avoir repris ses droits sur le site d'étude, mais le sol et le sous-sol restent encore très marqués et perturbés comme en témoigne le levé LiDAR et les prospections de terrain. Il serait intéressant de comparer cette dynamique de la végétation sur un site comparable pour voir si les mêmes dynamiques se reproduisent mais également sur un site où la forêt était déjà présente avant les ravages de la première guerre mondiale. Concernant l'étude des sols, il faudrait multiplier les relevés et la quantification des éléments métalliques tout en améliorant la quantification de ces derniers, car certains sont trop fins pour être observés à l'œil nu et tous ne sont pas magnétiques. Et enfin, il faudrait comprendre leur comportement dans le sol pour mieux mesurer leur impact sur la croissance des végétaux.

\section{BIBLIOGRAPHIE}

Amat J.-P., 1987, « Guerre et milieux naturels : les forêts meurtries de l'Est de la France, 70 ans après Verdun », Espace géographique, tome 16, n³, pp. 217-233.

Bausinger T., Bonnaire E. et Preuß J., 2007, « Exposure assessment of a burning ground for chemical ammunition on the Great War battlefields of Verdun. », Science of the Total Environment, 382, pp. 259-271.

Decencière Ferradière H., 1960, « Origine des forêts domaniales », Revue Forestière Française, $\mathrm{n}^{\circ} 4$, pp. 237-249.

Doerr S.H., Shakesby R.A., Walsh R.P.D., 2000, « Soil water repellency: its causes, characteristics and hydrogeomorphological significance ",Earth-Science Reviews, 51, pp. 33-65.

Doerr S.H., Shakesby R.A., Dekker L.W. et Ritsema C.J., 2006, « Occurrence prediction and hydrological effects of water repellency amongst major soil and land-use types in a humid temperate climate ", European Journal of Soil Science, 57, pp. 741-754.

Ghanbarizadeh L. et Nejad T.S., 2012, "The Uptake of Heavy Metals in Plants », Life Science Journal, 9(3), pp. 2310-2313.

Hupy J. et Schaetzl R., 2006, «Introducing « bombturbation », a singular type of soil disturbance and mixing ", Soil science, 171, 11, pp. 823-836.

Johnson J., Schewel L., Graedel T.E., 2006. « The contemporary anthropogenic chromium cycle. », Environmental Science \& Technology, 40, pp. 7060-7069.

Revue de géographie historique, 14-15 | 2019 
Jørgensen S.S. et Willems M., 1987, « The fate of lead in soils : The transformation of lead pellets in shooting-range soils ", Ambio, 16, pp. 11-15.

Leroux J. et Allouc J., 1988, « Structure de la Champagne crayeuse occidentale et de la Côte champenoise : mise en évidenceet analyse dedéformations anté- et post-thanétiennes ", Géologie de la France, 4.

Lewis J., Sjöström J., Skyllberg U. et Hägglund L., 2010. « Distribution, chemical speciation, and mobility of lead and antimony originating from small arms ammunition in a coarse-grained unsaturated surface sand "Journal of Environmental Quality, 39, pp. 863-870.

Lin Z., Comet B., Qvarfort U. et Herbert R., 1995, « The chemical and mineralogical behavior of Pb in shooting range soils from central Sweden », Environmental Pollution, 89 (3), pp. 303-309.

Manninen S. et Tanskanen N., 1993, « Transfer of lead from shotgun pellets to humus and three plant species in a Finnish shooting range », Archives of Environmental Contamination and Toxicology, 24, pp. 410-414.

Taborelli P., Devos A., Laratte S., Brenot J., Bollot N., Cancès B. et Desfossés Y., 2017, « Apport des plans directeurs et de l'outil LiDAR aéroporté pour la caractérisation des impacts morphologiques de la Grande Guerre : exemple de la cote 108 (Berry-au-Bac, France) », Géomorphologie : relief, processus, environnement, vol. 23, nº 2, pp.155-169.

Vasarevičius S. et Greičiūtė K., 2004, « Investigation of soil pollution with heavy metals in Lithuanian military grounds ", Environmental Engineering and Landscape Management, XII(4), p. 132

\section{RÉSUMÉS}

Le site d'étude de 180 hectares, communément appelé la « Cote 108 » se trouvait à l'interface des fronts français et allemand durant la Première Guerre mondiale. Il a été impacté par une guerre des mines dans sa partie ouest et par un intense pilonnage dans sa partie est. A la fin de la guerre, le site n'avait quasiment plus aucune végétation et était marqué par de nombreux entonnoirs.

Notre étude s'est intéressée à la recolonisation de la végétation sur cette zone dévastée par la Première Guerre mondiale. Pour cela, six séries de photographies aériennes (IGN) allant de 1929 à 2016 ont été étudiées afin de reconstituer la dynamique de recolonisation de la végétation dans le temps. Parallèlement, une étude du sol a été menée. Son but a été de décrire le sol de la zone d'étude et de quantifier les macro-restes métalliques subsistant encore aujourd'hui.

Cette étude a ainsi montré deux situations de recolonisation bien différentes : l'une spontanée, l'autre plantée et gérée par l'ONF. Malgré les plantations, la végétation a eu du mal à reprendre ses droits. Il a fallu 50 ans pour que la zone en recolonisation spontanée soit entièrement recouverte de végétation herbacée et arbustive et finalement un siècle pour assister à une recolonisation quasi-totale de la zone. Grâce aux descriptions de sol menées, nous avons pu proposer un ensemble de facteurs liés au sol et à la végétation pouvant expliquer cette lente recolonisation du site par la végétation.

The 180-hectare study site, commonly known as " Hill 108 », was at the interface of the French and German fronts during the First World War. It has been impacted by a mine war in its western part and by intense shelling in its eastern part. At the end of the war the site had almost no vegetation and is marked by many funnels. Our study focused on the re-colonization of vegetation in this region devastated by the First World War. To this end, six series of aerial photographs (IGN) from 1929 to 2016 were studied in order to reconstruct the dynamics of vegetation recolonization over time. At the same time, a soil survey was carried out. Its purpose 
was to describe the soil in the study area and to quantify the metal macro-remains that still exist today. This study highlighted two very different recolonization situations: one spontaneous, the other planted and managed by the ONF. Despite the plantations, the vegetation had difficulty recovering its rights. It took 50 years for the area under spontaneous recolonization to be completely covered with herbaceous and shrub vegetation and finally a century to attend almost complete recolonization of the area. Thanks to the soil descriptions conducted, we were able to propose a set of soil and vegetation factors that could explain this slow recolonization of the site by vegetation.

\section{INDEX}

Mots-clés : Recolonisation de la végétation, photo-interprétation, sol, restes métalliques

Keywords : Vegetation, recolonization of vegetation, photo-interpretation, soil, metal remains

\section{AUTEURS}

\section{EMILIE GALLET-MORON}

Ingénieur d'études, Plateforme Humanités Numériques - Plateau Cartographie Université de Picardie

\section{JÉRÔME BURIDANT}

Professeur des universités en géographie, Université de Picardie, Unité EDYSAN, Ecologie et Dynamique des Systèmes Anthropisés, UMR CNRS 7058)

\section{LAURENT CHALUMEAU}

Maître de conférences, Université de Picardie, Unité EDYSAN, Ecologie et Dynamique des Systèmes Anthropisés, UMR CNRS 7058 